\title{
AUTONOMOUS AIRCRAFT OPERATIONS USING RTCA GUIDELINES FOR AIRBORNE CONFLICT MANAGEMENT
}

\author{
Karthik Krishnamurthy, Titan Corporation, Hampton, VA \\ David J. Wing, Bryan E. Barmore, Richard Barhydt, Michael T. Palmer, Edward J. Johnson, \\ Mark G. Ballin, NASA Langley Research Center, Hampton, VA \\ Todd M. Eischeid, Booz Allen \& Hamilton, Hampton, VA
}

\begin{abstract}
A human-in-the-loop experiment was performed at the NASA Langley Research Center to study the feasibility of DAG-TM autonomous aircraft operations in highly constrained airspace. The airspace was constrained by a pair of special-use airspace (SUA) regions on either side of the pilot's planned route. Traffic flow management (TFM) constraints were imposed as a required time of arrival and crossing altitude at an en route fix. Key guidelines from the RTCA Airborne Conflict Management (ACM) concept were applied to autonomous aircraft operations for this experiment. These concepts included the RTCA ACM definitions of distinct conflict detection and collision avoidance zones, and the use of a graded system of conflict alerts for the flight crew.
\end{abstract}

Three studies were conducted in the course of the experiment. The first study investigated the effect of hazard proximity upon pilot ability to meet constraints and solve conflict situations. The second study investigated pilot use of the airborne tools when faced with an unexpected loss of separation (LOS). The third study explored pilot interactions in an over-constrained conflict situation, with and without priority rules dictating who should move first.

Detailed results from these studies were presented at the $5^{\text {th }}$ USA/Europe Air Traffic Management R\&D Seminar (ATM2003). This overview paper focuses on the integration of the RTCA ACM concept into autonomous aircraft operations in highly constrained situations, and provides an overview of the results presented at the ATM2003 seminar. These results, together with previously reported studies, continue to support the feasibility of autonomous aircraft operations.

\section{Introduction}

NASA's Advanced Air Transportation Technologies project is developing a far-term concept of operations for Air Traffic Management (ATM). This concept of operations, called Distributed Air/Ground Traffic Management (DAG TM) [1], is NASA's vehicle for researching and developing the RTCA Free Flight concept [2], wherein flight crews select their path and speed in real time while conforming to restrictions established for safety and flow management.

\section{Autonomous Aircraft Operations}

DAG-TM Concept Element 5 (CE-5) [3] specifically defines operations in the en-route and terminal-transition domains of flight, and it proposes the establishment of a new category of flight operations: autonomous flight rules (AFR). According to the CE-5 concept, an AFR aircraft would generally operate in the same airspace as existing instrument flight rules (IFR) aircraft, but the AFR flight crew would retain a distinct set of authorities and responsibilities. Trained flight crews of AFRequipped aircraft are given the authority to dynamically plan and execute their preferred 3D trajectories without coordinating with the groundbased air traffic service (ATS) provider. With this authority comes full responsibility for traffic separation and conformance to operational constraints; the ATS provider establishes these constraints in order to safeguard special-use airspace and manage traffic flows into high-demand terminal areas. Under normal operations, the ATS provider is neither required nor expected to intervene in AFR operations throughout the en-route and terminaltransition domains. However, the ATS provider continues to provide traditional IFR services to nonautonomous ("managed") aircraft. It is anticipated that AFR operations would provide airspace users a 
significant degree of flexibility to operate costeffectively, and would enable the airspace system to accommodate a substantial increase in traffic volume over that manageable by a ground-based IFR system. This scalability would presumably result from minimizing the interactions between autonomous aircraft operations and the ATS provider.

The concept confines interactions between the ATS provider and autonomous aircraft under nominal conditions to traffic flow management (TFM). In the DAG-TM concept, time-based arrival metering will be the principal TFM tool. Using predictive information on arrivals and airspace status, the ATS provider establishes flow metering by issuing required-time-of-arrival (RTA) clearances and crossing restrictions at metering fixes to AFR aircraft. Once these clearances and restrictions are received and accepted by the flight crew, the interaction between the ATS provider and this autonomous aircraft is minimized until the aircraft crosses the fix.

Flight crews of autonomous aircraft are assisted in fulfilling their AFR responsibilities by flight deck tools and displays integrated with the onboard avionics system. NASA Langley Research Center is developing a research prototype of this toolset, the Autonomous Operations Planner (AOP). AOP is being developed $[4,5]$ to integrate crew preferences, TFM constraints, airspace constraints and traffic information into the planning and execution of efficient autonomous operations.

\section{RTCA Airborne Conflict Management Concept}

Concurrent to the development of AOP, Working Group 1 of RTCA Special Committee 186 has developed an operational concept [6] for Airborne Conflict Management. The ACM concept includes three distinct functions: conflict detection (CD), conflict prevention (CP), and conflict resolution (CR). As envisaged by RTCA, the CD function will allow long-range detection of conflicts, providing flight crews the time to develop and implement an optimal solution to the conflict. The CP function will predict conflicts that could be caused by changes to current ownship state and intent. The CR function will provide guidance cues or resolution trajectories to solve existing conflicts.
The RTCA ACM concept also features a pair of cylindrical airspace zones surrounding each aircraft (Figure 1). The outer zone (Conflict Detection Zone, or CDZ) reflects standard legal separation minima, while the significantly smaller inner zone (Collision Avoidance Zone, or CAZ) forms a tight "near miss" envelope around the aircraft. In the ACM concept, the CD function predicts penetrations of both these zones. Flight crews are alerted to conflicts, defined as predicted penetrations of the $\mathrm{CDZ}$, so that they can avoid violating legal separation minima. They are also alerted to predicted CAZ penetrations so that they can avert near miss situations.

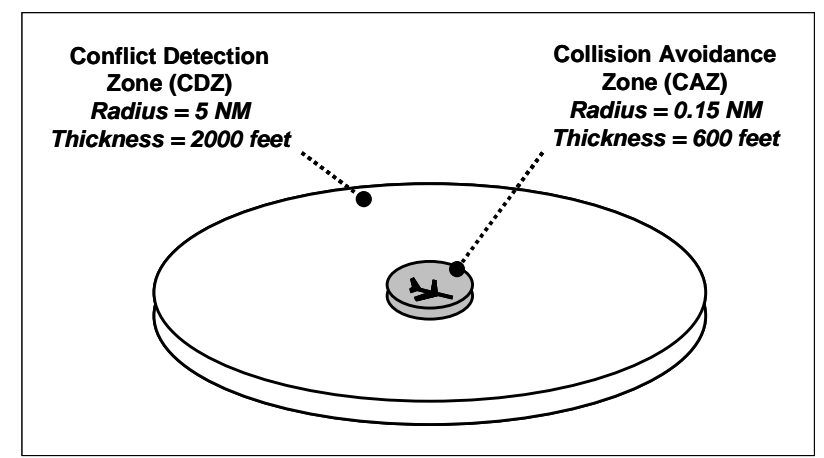

Figure 1. Conflict Detection And Collision Avoidance Zones (Not To Scale)

Three graded CD alert levels are recommended by the RTCA ACM concept. A "Low Level Alert" indicates the presence of a conflict (i.e., predicted penetration of the CDZ or loss of separation) that is sufficiently distant to allow the flight crew the option of developing and implementing optimized resolutions. A "CDZ Alert" is issued if the conflict is not resolved prior to a defined time threshold, to indicate that the conflict is proximate and that prompt resolution action is required. The ACM system also scans for possible penetrations of the CAZ. If a near-term CAZ penetration is predicted, a "CAZ Alert" is issued, indicating to the crew that immediate action is required to avert a possible collision or near miss situation. A CAZ alert is timed to precede the issuance of a Traffic Alert and Collision Avoidance System (TCAS) Resolution Advisory. ${ }^{1}$

\footnotetext{
${ }^{1}$ In the RTCA concept, the CR function of the ACM system will be completely interoperable with, but functionally independent of, existing Airborne Collision Avoidance Systems such as TCAS II.
} 


\section{Experiment Objectives}

Autonomous aircraft operations in DAG-TM are consistent with many precepts of the RTCA ACM concept, allowing key guidelines from the RTCA concept to be integrated into the DAG-TM tools and procedures. The prototype AOP toolset has been designed to perform the three ACM functions of CD, CP and CR. AOP uses state and intent (FMS flight plan) information from ownship and traffic aircraft to perform $\mathrm{CD}$, and to provide both strategic and tactical CR to solve intent-based and state-based conflicts respectively. It also provides CP guidance using the state-based Predictive Airborne Separation Assurance System (P-ASAS) concept pioneered by the NLR [7]. Introducing the concepts of CDZ and CAZ into AOP enhances its capability to support all ACM functions. In addition, introducing into AOP the graded system of alerts outlined by RTCA provides a simple and intuitive framework for crew alerting to conflicts. The study presented here tested the integration of these key features of the RTCA ACM concept into AOP, and assessed pilot use of these ACM functions, with the overall objective of exploring the feasibility of autonomous aircraft operations.

The fundamental challenge of autonomous aircraft operations is ensuring that flight crews of autonomous aircraft, upon receiving and accepting TFM constraints, are able to plan and execute efficient conflict-free trajectories that meet all TFM constraints within established tolerances. In currentday operations, air traffic controllers perform similar tasks for all aircraft within their sectors. Flight crews of autonomous aircraft, however, have to deal with only those conflicts that involve their own aircraft; that is, they need to solve only a subset of all conflicts in the airspace, thereby reducing conflict management workload to a linear function. Further, the introduction of ADS-B provides longrange surveillance, and hence long look-ahead periods are available for conflict detection. With fewer aircraft to consider for separation assurance, greater time and flexibility to solve conflicts, and the ability to monitor developing situations more closely, it is hypothesized that flight crews of autonomous aircraft may indeed be able to readily manage autonomous flight through nominal environments, and do so in significant traffic densities.
A variety of research studies support this hypothesis. Numerous approaches to providing both unconstrained $[7,8]$ and TFM-constrained $[9,10]$ airborne separation assurance have been developed and successfully tested in nominal conditions over the past several years. In an earlier piloted simulation using the AOP prototype, the authors studied tactical and strategic modes for autonomous aircraft operations with TFM constraints, and found that both operational modes are consistent with, and support feasibility of, autonomous aircraft operations at up to three times current traffic density [10].

However, in order to fully explore autonomous aircraft operations and establish the limits of feasibility of the underlying concept, it is necessary to study more challenging situations involving highly constrained and off-nominal conflict scenarios. Investigations of such situations provide better insight into concept feasibility and concept robustness, while providing guidelines for the continued development of airside ACM tools and groundside approaches to TFM that minimize airground interactions in autonomous aircraft operations.

A highly constrained situation relevant to concept feasibility is one where airspace hazards, traffic flows and TFM constraints all co-exist to pose a simultaneous challenge to autonomous aircraft operations. Several research questions can be addressed in these challenging situations. For example, what is the effect of the proximity of hazards and traffic on the ability of autonomous aircraft to successfully satisfy all constraints? Can conflicts in these situations be resolved without additional air/ground interaction? Off-nominal situations are also of interest, including cases where the full nominal sequence of alerts is not presented to the pilot due to ADS-B range limitations, improper maneuvering by another pilot, emergency maneuvering by another pilot, etc. How would pilots respond to the resulting near-term "pop-up" conflicts? Another off-nominal situation of import probes the interdependence of conflict avoidance and TFM constraints. What if it were literally impossible for all constraints to be simultaneously met - will safety be compromised if pilots are unaware of the over-constrained nature of the situation?

The experiment reported herein addressed these issues in a human-in-the-loop simulation of 
autonomous aircraft operations. The exploratory study had three specific objectives: (a) to determine the effect of varying hazard proximity upon pilot ability to maintain separation assurance and adhere to TFM constraints; (b) to investigate pilot use of AOP in near-term, pop-up conflicts; and (c) to determine the nature of pilot interactions in an overconstrained conflict situation. Investigations into objectives (a) and (b) above included a preliminary investigation of the effect of lateral separation criteria upon pilot use of the prototype ACM system. Numerous other factors would have to be explored to fully evaluate whether separation criteria could be modified, and making a case for adopting a smaller separation criterion is well outside the scope of this study. Investigations into objective (c) of the study also probed the need for and effectiveness of priority flight rules in preserving traffic separation in the over-constrained conflict situation. For this portion of the study, priority rules were integrated into the alerting scheme that was implemented in AOP. In total, the three objectives allowed a probing of the limits of concept feasibility and an assessment of the effectiveness and pilot interaction with a prototype decision support tool with capabilities based on the RTCA ACM concept.

\section{Experimental Approach}

The experiment was performed in the Air Traffic Operations Laboratory (ATOL) at the NASA Langley Research Center. The ATOL is a distributed simulation environment where pilots of multiple simulated aircraft can interact in preplanned or dynamically developing scenarios. As described earlier, features of the RTCA ACM concept were integrated into ATOL aircraft simulators for the experiment.

As part of this integration, AOP (as described in [4]) was modified in several ways. CD was expanded to perform state-based CD on the CAZ. The alerting scheme by which AOP alerts the crew to conflicts was modified to use the graded alert levels recommended by RTCA. The time-lines for the alerts were selected in accordance with the ranges recommended by RTCA (Figure 2).

In addition, the CP system in AOP (based on NLR's P-ASAS concept) was extended to provide preventive information on CAZ avoidance. While not a specific recommendation in the RTCA ACM concept, this capability was tested as a logical extension to the nominal CP system. The resulting CP system provides three levels of $\mathrm{CP}$ guidance. The time-horizons for these three levels of $\mathrm{CP}$ were set to 1 minute (red band, indicating collision prevention), 2 minutes (amber band, indicating “do not fly” zone), and 5 minutes (cyan band, indicating "proceed with caution”).

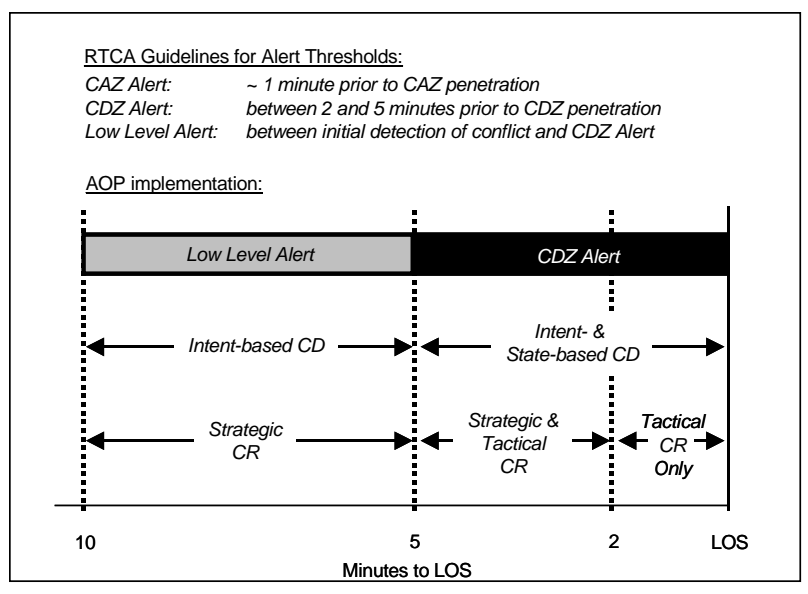

Figure 2. Implementation Of RTCA Guidelines

Where the ACM concept is silent on the issue of what traffic may be of interest to the crew, the ATOL implementation assigned an additional "point-out" alert level for "possible threat" aircraft. These were traffic aircraft that were not in conflict but could still be of interest to the crew, either due to being proximate, or being within easy range of a conflict. Traffic aircraft not qualifying for one of these four alerts were not displayed.

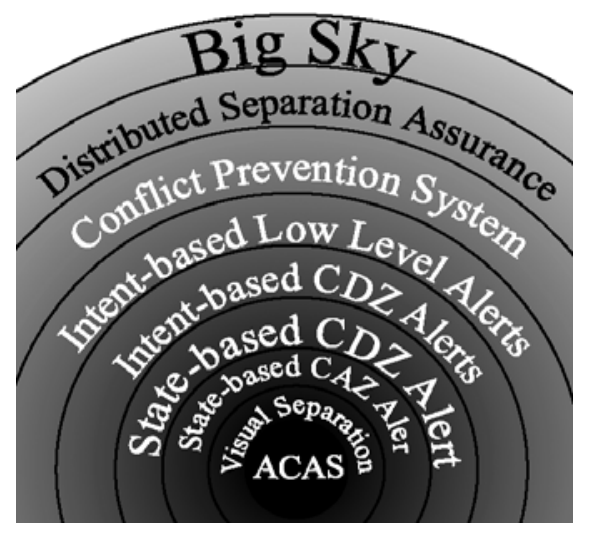

\section{Figure 3. Multiple Layers Of Protection}

With the integration of RTCA guidelines into AOP, multiple layers of protection are now provided for separation assurance in autonomous 
aircraft operations (Figure 3). Simulation studies of Free Flight in en route airspace [11] have suggested that the likelihood of conflicts is rather low (i.e., big sky), and if conflicts do arise, CE-5 introduces redundancy in conflict management by distributing the responsibility for separation assurance. The ACM toolset then provides flight crews with several additional layers of protection before they enter the realm of visual separation assurance and ACAS: $\mathrm{CP}$ tools help prevent the creation of new conflicts; Low Level Alerts based on intent and state information can detect conflicts early and reduce missed alerts; CDZ Alerts introduce a measure of urgency by prompting pilots to take timely avoidance actions; and CAZ Alerts provide pilots with additional time to react to potential collision situations.

\section{Experiment Design}

Sixteen currently or recently active airline pilots participated in the experiment, which was conducted over a 2-week period in the Fall of 2002. Only the autonomous aircraft operations aspect of CE-5 was represented in the simulation; the ATS provider and piloted managed aircraft were not simulated, nor were they needed to achieve the experimental objectives. The pilots were spread among four groups, which flew in the simulation one group at a time. Efforts were taken to disguise the fact that pairs of them would occasionally interact through traffic conflicts. The majority of conflicts were scripted with automated aircraft.

The pilots received comprehensive training on the procedures, alerting levels, and conflict management tools through printed materials, classroom briefings, and hands-on practice with one-on-one instruction before they flew the planned test scenarios. Additionally, the first test scenario after training was in fact a buffer scenario, unbeknownst to the pilots, to verify the required pilot proficiency level had been reached. Including this buffer scenario, each pilot flew a total of 10 scenarios, counterbalanced for order, each based on the same basic scenario design described below.

\section{Experiment Scenarios}

The basic experimental scenario consisted of an en-route trajectory constrained by special-use airspace. The sizes and shapes of these SUAs were realistic, motivated by a current airspace configuration in the area of Reno, Nevada, USA. An autonomous aircraft, controlled in the experiment by a single human pilot, was established on a flight plan between the SUAs [Figure 4(a)]. In addition to the subject-piloted aircraft, the airspace was populated with other aircraft traveling in both directions through the corridor at altitudes above, below, and equal to that of the subject pilot's aircraft. This traffic provided a realistic and distracting environment for the subject pilot. Note that a common structured route through the corridor was not used for any autonomous aircraft in the scenario.

To meet hypothetical TFM needs (details not included or required in the simulation), the flight plan of the aircraft included a waypoint with a required time of arrival (RTA) and a "cross at" altitude restriction, both of which were supposedly assigned by the ATS provider. These constraints were pre-entered in the Flight Management System (FMS) flight plan at the outset of the simulation, which began approximately 200 miles (25 minutes) before the RTA waypoint. These constraints provide quantifiable metrics against which the effectiveness of the cockpit decision tools and displays could be evaluated, and also provide mission goals for the subject pilots. Crossing the RTA waypoint within \pm 30 seconds of the assigned time, within \pm 500 feet of the assigned altitude, and within \pm 2.5 NM laterally was defined to be acceptable conformance to the TFM constraints. The pilots were briefed on these tolerances.

Subject pilots were instructed to maintain traffic separation as a top priority, and to achieve the assigned waypoint constraints as a second priority. If the pilot determined that any of the assigned constraints could not be met for any reason, the instructions were to "notify" the ATS provider as early as possible, using three labeled buttons provided in the flight simulator for this purpose. Pilots were not constrained to follow the initial flight plan. Rather, they were advised to make their own best judgments regarding the conduct of the flight. The pilots were free to choose the lateral and vertical path that they felt best met their objectives. Flights were not constrained to the hemispherical altitude flight levels in accordance with the CE-5 concept. Therefore, it was possible for subject pilots to encounter unplanned conflicts and flight situations. 
This basic scenario was modified as required to investigate the three main research objectives. Research issues common to all scenarios included safety of flight operations through the corridor, acceptability and usability of the cockpit tools, and the pilot use of combined strategic and tactical resolution tools.

\section{Hazard Proximity Scenarios}

The primary research issues for this part of the study were the interactive effects of required lateral separation and constrained airspace on pilot ability to maintain separation and meet assigned constraints. The independent variables studied were the width of the SUA "corridor" and the lateral dimensions of the CDZ surrounding each aircraft. In addition, unbeknownst to the subject pilot, there was one planned conflict, with separation predicted to be lost approximately halfway through the corridor.

The basic scenario was studied with two corridor width conditions: a narrow corridor and a wide corridor [Figure 4(b)]. Each subject pilot flew both conditions as part of the within-subjects experiment design. The narrow corridor had a minimum width of approximately $33 \mathrm{~nm}$. The wide corridor had a minimum width of approximately $65 \mathrm{~nm}$. For data collection flights through the wide corridor, additional traffic aircraft were added to the simulation to maintain the aircraft density and, hence, the difficulty of the conflict avoidance task.

The second independent variable studied was the lateral dimension of the CDZ. Again, two conditions were studied: a CDZ with a $3 \mathrm{NM}$ radius and a CDZ with a 5 NM radius. Each data collection flight involved only one of these conditions. Subject pilots were briefed on the CDZ size in effect prior to each flight, and the AOP algorithms were adjusted to the appropriate size of the CDZ. In all cases, the vertical dimension of the CDZ was \pm 1000 feet.

Each pilot flew all combinations of the 2x2 matrix of corridor width and separation criteria, for a total of four hazard proximity runs per pilot. Data from five runs was lost to simulation faults, resulting in a total of 59 valid hazard proximity runs for data analysis.

\section{Pop-up Conflict Scenarios}

This portion of the study was intended to evaluate the effectiveness of the prototype ACM tools in near-term conflict situations that could be caused by, for example, an aircraft that is maneuvering improperly in response to a conflict alert, or an aircraft forced to descend rapidly due to some emergency. In addition, the study compared the effect of 3 and $5 \mathrm{NM}$ lateral separation standards (with $1000 \mathrm{ft}$ vertical separation) on pilot ability to safely resolve near-term traffic conflicts and regain unexpectedly lost separation.

The basic scenario of Figure 4 (a) was used for this portion of the study. In order to simulate an unexpected near-term conflict, a scripted intruder aircraft was maintained on a track parallel and coaltitude to the subject piloted aircraft until a predetermined time in the scenario. However, this intruder was "hidden" from the subject pilot by suppressing its ADS-B broadcast. This simulation procedure was necessary to create the unexpected pop-up event.

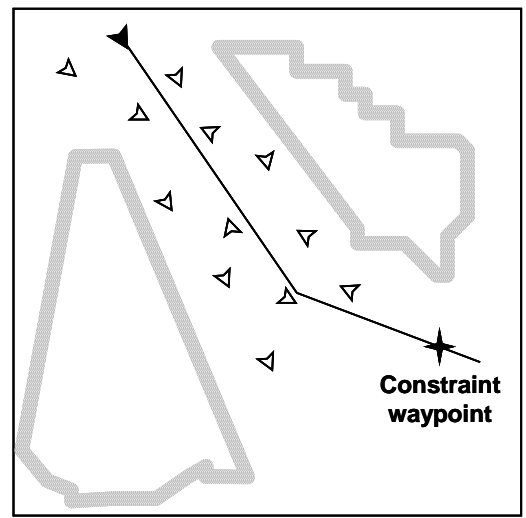

(a) Basic Scenario

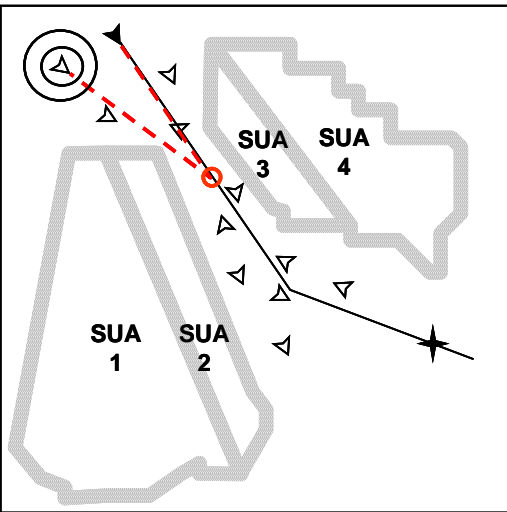

(b) Hazard Proximity Scenario

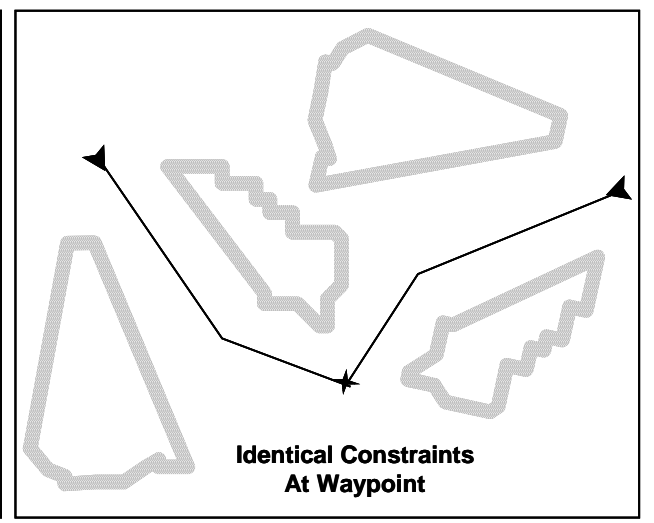

(c) Over-Constrained Scenario

Figure 4. Experiment Scenarios 
At the appropriate time, generally as the subject aircraft was emerging from the SUA corridor, the simulation manager turned the intruder toward the subject aircraft and activated the intruder's ADS-B broadcast. As a result, the intruder would "pop-up" about 6 NM away from the subject aircraft, on a conflicting heading and at the same altitude. Due to the proximity of the intruder at first appearance, separation would almost certainly be lost and immediate maneuvering would be required to regain required separation. The same conflict geometry (location, approach angle, and time to closest approach) was used for both $3 \mathrm{NM}$ and 5 NM separation zone cases. This allowed a comparison of risk incurred and highlighting of any variation in pilot performance due to an aircraft being inside or outside the CDZ.

Each pilot flew two runs with pop-up conflicts. Of the resulting 32 runs, four runs were marred by a simulation failure late in the run. Data on the popup conflict and consequent pilot responses were not contaminated in these four runs, and were included in subsequent data analysis. However, the constraint conformance data were not useable.

\section{Over-Constrained Conflict Scenarios}

The scenarios used to investigate this objective were identical to the basic scenario of Figure 4(a), except that the flight plans of two subject-piloted aircraft intersected at the constraint waypoint, which could be considered a metering fix ${ }^{2}$. The over-constrained conflict was created by issuing identical crossing constraints (RTA and altitude) at that waypoint to both aircraft [Figure 4(c)]. Of course, a significant failure at several levels of the NAS must have occurred to permit this situation to arise at all; nevertheless, the objective of this study was specifically to determine how the airborne side might react to such potentially hazardous situations in an effort to test the robustness of distributed autonomous aircraft operations.

Efforts were taken to ensure that both pilots were not aware a priori of the nature of this conflict, nor that they would be interacting with another subject pilot in this scenario. Each pilot pair encountered this scenario three times. For two of these

\footnotetext{
${ }^{2}$ Descents were not included in the scenario because proper FMS descent capability in the simulation had not yet been completed.
}

runs priority rules (governing which aircraft should move first) were in effect. The two runs were conducted so that each pilot had a turn at higher and lower priority, to counterbalance the test matrix. Priority was not actively indicated to the pilots; rather a passive technique was used in that the alert level (i.e. point out, Low Level Alert, CDZ Alert) was staggered in time to favor the higher priority aircraft, until an established time threshold was reached when both aircraft received CDZ Alerts (Figure 5). The third run was performed with no priority rules; that is, both pilots received alerts at the same time (as was indicated in Figure 2). Comparison of the data from these runs provides insight into the effectiveness of implicit coordination (through priority rules) in resolving this challenging situation.

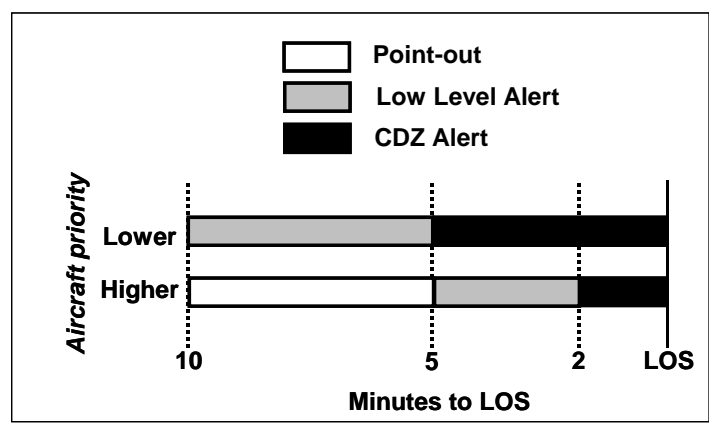

\section{Figure 5. Implementation Of Priority Through Staggered Alerting Thresholds}

Of the 48 data runs conducted to investigate these issues, four runs were lost to simulation faults. The remaining 44 runs were analyzed in pairs (since pilots interacted in pairs in the course of this scenario). Of these 22 pairs of runs, 10 were conducted without priority rules in effect, and 12 had priority rules in effect.

\section{Results And Discussion}

Results from these studies were previously reported at the $5^{\text {th }}$ USA/Europe ATM R\&D Seminar (ATM2003) [12-14]. This overview paper focuses on the integration of the RTCA ACM concept into autonomous aircraft operations in highly constrained situations, and provides an overview of the results presented at the ATM2003 seminar. 


\section{Safety Of Flight Operations}

A fundamental indication of whether safety was compromised in the course of experiment scenarios is the occurrence of loss of separation events. Except for the scripted pop-up conflicts, pilots were expected to receive sufficient alerts ahead of time to be able to use the ACM tools and solve the conflict. However, a few LOS occurrences were observed in the course of the hazard proximity and overconstrained runs.

Not counting simulation faults, pilots lost separation twice in the hazard proximity runs. One LOS situation resulted from a subject-pilot initiating a climb without proper consultation of CP information presented on the Primary Flight Display (which warned him against the climb). The other LOS situation occurred when a pilot initiated a vertical maneuver without making certain that required lateral separation existed with traffic aircraft ${ }^{3}$.

In the over-constrained runs, there was one LOS occurrence not due to a simulation fault. The two subject-piloted aircraft lost separation close to the RTA waypoint when one of the pilots climbed prematurely after passing the other aircraft, without actively ensuring that lateral separation with the other aircraft existed, again ignoring vertical CP information.

Closer examination of these three LOS situations indicates that none of them represented a significant safety risk; rather, they were in the nature of minor infractions. These LOS situations, however, do point to areas of potential improvement in pilot training and the display of $\mathrm{CP}$ information. The low incidence of LOS situations overall indicate that pilots were largely successful in maintaining safe flight in the course of these scenarios.

\section{Overall Constraint Conformance}

As was stated earlier, a major challenge in autonomous aircraft operations is the ability of flight crews to successfully plan and execute conflict-free paths that meet all TFM constraints. Data collected in the course of the hazard proximity and

\footnotetext{
${ }^{3}$ The pilot failed to use a display feature that would have displayed a circle on the Nav Display indicating the traffic's separation zone.
}

pop-up conflict scenarios indicate that pilots were largely successful in performing these tasks. The over-constrained conflict scenarios, as stated earlier, were designed such that both pilots could not simultaneously meet the TFM constraints. Figure 6 indicates the observed deviations from assigned constraints in the course of all runs employing the first two scenario types.

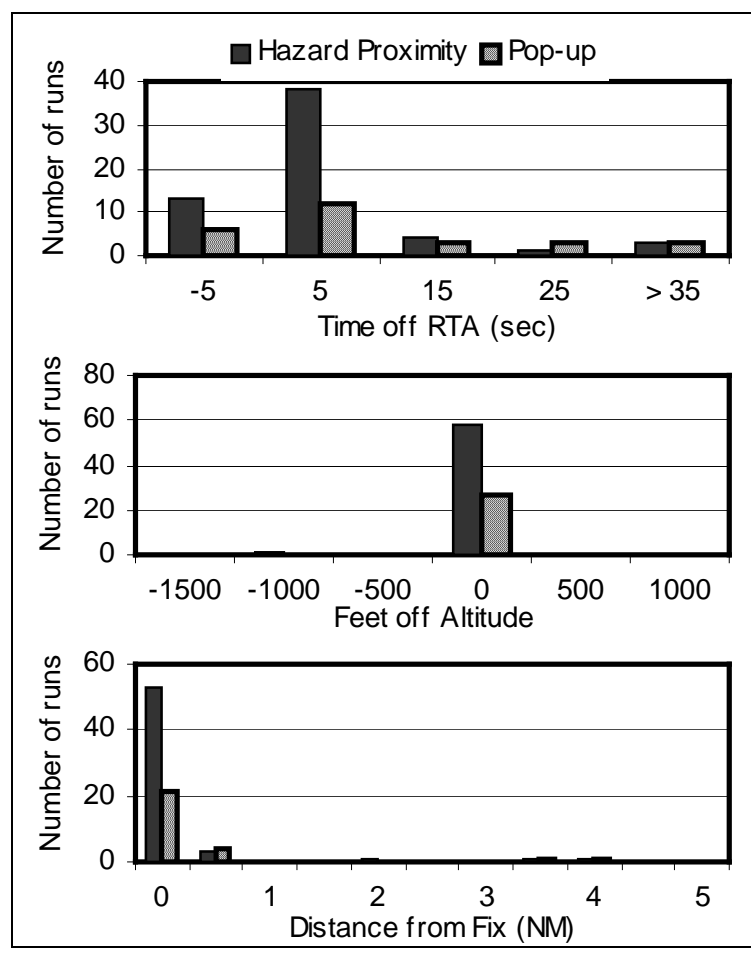

\section{Figure 6. Deviations From Assigned TFM Constraints}

It is clear that an overwhelming majority of pilots successfully met all TFM constraints in these scenarios. Further, a majority of pilots met their constraints well within permitted tolerances. This behavior is true even in the runs featuring pop-up conflicts, where sudden conflicts appeared about midway through the run. These results support feasibility of autonomous operations even under highly constrained and off-nominal situations.

\section{Pilot opinions on alerting scheme}

Following the end of each data run, pilots were asked to fill out a questionnaire that included the following question: "How intuitive was the conflict alerting system? An intuitive alerting system would provide clear information about which aircraft you 
were in conflict with and the urgency of the conflict." The ordinal rating scale ranged from "not at all intuitive" (1) through "neutral” (4) to "very intuitive" (7). Pilot opinions across all run types are presented in Figure 7.

The data indicate that mean responses were above neutral for all run types: 5.85 for hazard proximity runs, 4.96 for pop-up conflict runs, and 5.52 for over-constrained runs. Of the 130 runs for which data is presented here, pilots in 110 runs (85\%) rated the alerting system to be above neutral, compared to just 11 runs (8.5\%) where pilots rated the system to be below neutral. These results indicate that pilots found the implementation of the RTCA ACM alerting concept to be useful and intuitive in performing autonomous aircraft operations.

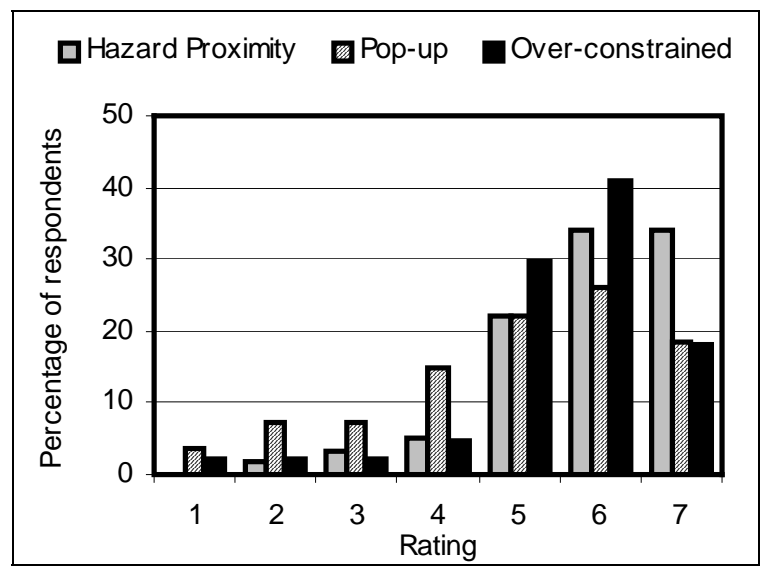

Figure 7. Pilot Opinions On Intuitiveness Of Alerting

\section{Hazard Proximity}

The objective of this part of the experiment was to investigate the effect of hazard and traffic proximity on the ability of autonomous aircraft to successfully satisfy all constraints. Overall results [12] from this part of the experiment indicate that airborne separation is feasible even under the tight airspace and arrival constraints, and that efficiencies are gained with a reduction of the lateral separation standard. The graded system of ACM alerts was effective in enabling both strategic and tactical management of conflicts within the operational constraints defined by the scenario.

Stability of the overall traffic situation can be augmented if conflict resolutions have minimal impact on other aircraft in the vicinity. In all scenarios used in this experiment, special care was taken to ensure that there would be no other conflicts except the one planned along the original flight plan. Therefore, any additional conflicts that occurred during a scenario were due to the pilot deviating from the flight plan to avoid the planned conflict. These "second generation" conflicts constitute a ripple effect of the original conflict resolution. Data collected in the hazard proximity scenarios were analyzed for occurrences of secondgeneration conflicts.

Figure 8 shows the distribution of secondgeneration conflicts across pilot strategies to resolve the conflict in these scenarios. Here "strategic" resolutions indicate the pilot remained in FMS guidance throughout the resolution. "Modified strategic" resolutions are those where the pilot initially accepted a strategic resolution, but chose to modify it with a subsequent maneuver. "Tactical" resolutions indicate maneuvers using the Flight Control Panel and not the FMS. It is seen that pure-strategic resolutions caused no additional conflicts. This is to be expected, since strategic resolutions offered by AOP are designed to be conflict free for the next 20 minutes, which typically approached the length of the remaining scenario. However, tactical resolutions do not take into account other aircraft besides the conflicting aircraft, and the pilot was responsible for avoiding future conflicts through judicious use of the CP system. Further, for the pilots using the modified strategic resolution method, all second generation conflicts occurred after the tactical modification. These findings suggest that enhancing the tactical resolution system to take into account other aircraft would reduce conflict proliferation.

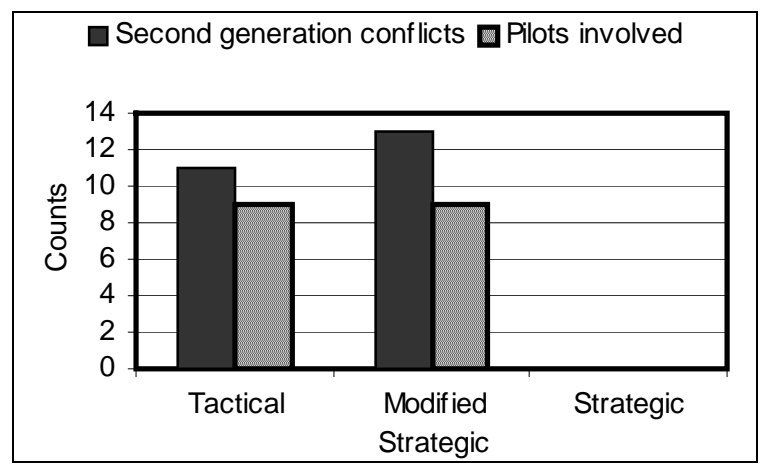

Figure 8. Second-Generation Conflicts 
Further analysis [12] of the data indicated that both strategic and tactical maneuver choices triggered an alert for second-generation conflicts. This analysis points to the need to provide CD on all provisional "what-if" trajectories; modifications to AOP are in progress to accomplish this function. It also motivates closer examination of what lookahead times are appropriate for conflict proliferation avoidance.

\section{Pop-up Conflicts}

The motivation for this part of the experiment was to assess pilot use of the ACM tools in responding to a near-term conflict alert, and to investigate the effect of reducing the lateral separation standard on pilot task of regaining separation.

Analysis of the data collected during these scenarios used a non-dimensional parameter, epsilon [15], as an indicator of threat severity. Dimensional quantities such as distance at closest point of approach do not reflect the unequal lateral and vertical extent of current-day separation standards. Epsilon normalizes the lateral and vertical components of distance between aircraft to the lateral and vertical extent of the CDZ. In order to compare results from 3 and 5 NM scenarios, all results using epsilon were calculated relative to a 5 NM standard. Analysis of the data [13] determined that compliance with the tactical guidance provided by the ACM tools reduced severity of the threat as indicated by higher epsilon values.

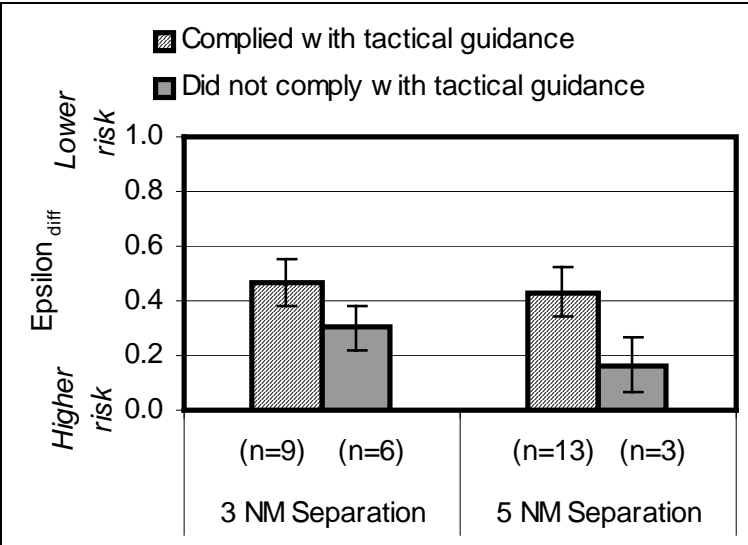

Note: Error bars represent 1 standard error of the mean.

Figure 9. Risk Mitigation Using ACM Tools
Analysis also focused on risk mitigation by pilot actions following the conflict alert. This analysis compared the epsilon value predicted at first alert appearance with the minimum epsilon actually achieved by the pilots' maneuvers. Large differences between these quantities indicate better risk mitigation by the pilot. Figure 9 indicates the findings of this analysis. It is seen that compliance with ACM tool guidance provided better risk mitigation for both 3 and 5 NM separation standards. However, the change in the lateral standard caused no significant difference in risk mitigation.

\section{Over-Constrained Conflicts}

The objective of this part of the experiment was to study pilot interactions in an overconstrained conflict situation, and to evaluate the usefulness of implicit coordination through priority rules in the resolution to this conflict. Results from the study [14] indicated that conflicts were safely resolved, both with and without priority rules. The following discussion focuses on the effect of priority rules upon constraint conformance among the pilot pairs.

The constraint conformance of the subject aircraft across all over-constrained data runs is presented in Figure 10. Three comparisons are presented: (1) all subject-aircraft with and without priority flight rules in effect; (2) subject-aircraft approaching from the right-hand (RH) and left-hand (LH) directions when priority flight rules were not in effect; and (3) subject-aircraft with and without priority when flight rules were in effect.

These results (1) indicate that approximately one-third of the aircraft met all of the waypoint constraints (time, position, and altitude) within acceptable tolerances, regardless of whether the priority flight rule system was used. When priority flight rules were in effect (3), aircraft that met all constraints were always the higher priority aircraft, indicating that the use of priority flight rules increases predictability regarding which aircraft in a pair is likely to prevail. This data also indicates the implementation of priority flight rules through staggered alerting thresholds was completely effective. Interestingly, when priority flight rules were not used (2), the RH aircraft in a conflict pair met their constraints about 2.5 times as frequently as the $\mathrm{LH}$ aircraft, suggesting that pilots may have subcon- 
sciously applied previously-learned standards for priority (such as VFR priority rules).

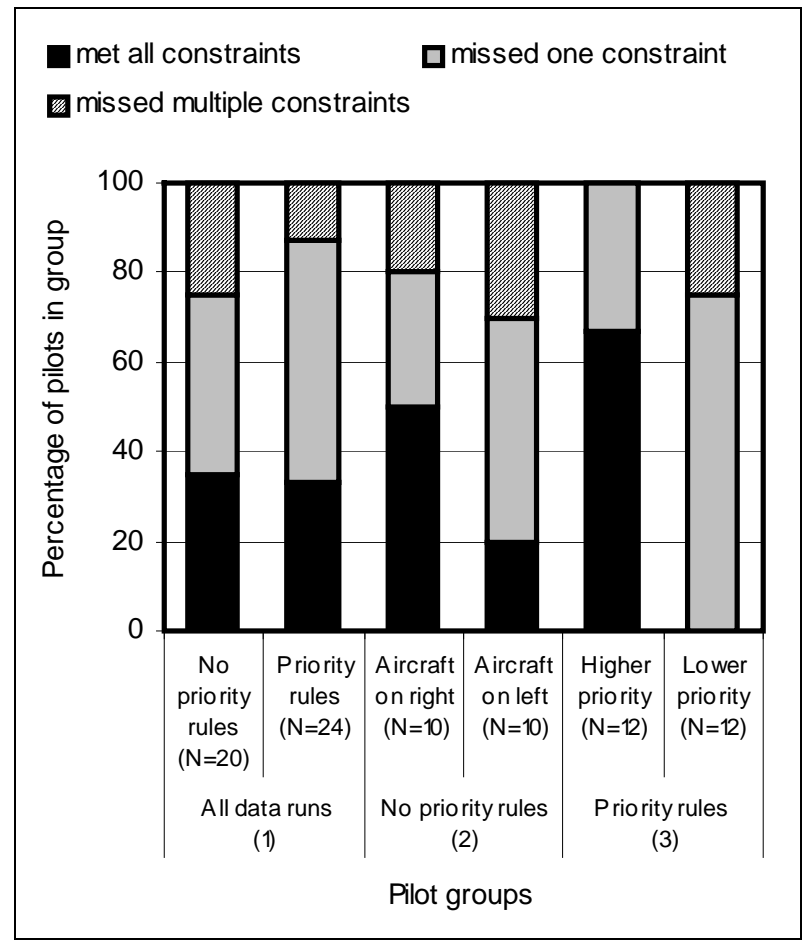

Figure 10. Constraint Conformance In OverConstrained Scenarios

Closer examination of these data brings into focus some important issues regarding broadcast intent. With priority flight rules in effect (3), in only two-thirds of the cases did one aircraft of the pair (the higher-priority aircraft) meet all constraints. The expectation was that all pilots with priority should have met all constraints. An investigation of the conflict alerting revealed that, in three of the four cases where the higher-priority (HP) aircraft did not meet all constraints, the HP aircraft received a conflict alert after the lower-priority (LP) aircraft had already implemented a strategic resolution. The lateral path-stretch in these strategic resolutions should have created an arrival delay that would have eliminated the conflict at the RTA waypoint. The conflict was still registered, however, because the LP aircraft were broadcasting their required time of arrival at the waypoint, rather than their estimated time of arrival. These data illustrate a hazard of broadcasting unachievable flight plan constraints, essentially false intent, in place of true trajectory predictions. Neighboring aircraft may use this false information to make maneuver decisions that, at a minimum, disrupt flight efficiency but may also lead to new conflicts. Broadcasting the commanded trajectory as the intent message, i.e., the four-dimensional path the autoflight system will actually command (assuming no further pilot inputs), would reduce or eliminate the hazards associated with disseminating false information. In fact, a recent update to the ADS-B system performance standards recommends the broadcast of commanded trajectory information [16].

\section{Conclusions And Future Work}

The feasibility of autonomous aircraft operations in highly constrained airspace was studied using a multi-piloted simulation. Pilots were tasked with negotiating an airspace corridor in the presence of traffic, and were required to meet TFM constraints at a waypoint beyond the corridor. As part of the experiment, RTCA guidelines for Airborne Conflict Management were integrated into a prototype flight deck tool to assist the pilot in performing $\mathrm{CD}, \mathrm{CP}$ and $\mathrm{CR}$ functions. The experiment yielded the following conclusions:

- AFR operations were feasible under the tight airspace and arrival constraints featured in this experiment. Pilots found the ACM alerting to be intuitive, even in scenarios featuring pop-up conflicts. The graded system of alerts was effective in enabling strategic and tactical conflict management. Safety of operations was not compromised by the combination of constraints.

- Reducing the lateral separation standard had no adverse effect on pilot ability to meet TFM constraints, and reduced the incidence of second-generation conflicts. In near-term conflicts, pilot ability to reduce threat proximity and mitigate risk appeared to depend more on compliance with the tactical resolution guidance than on CDZ size.

- The use of priority rules improved predictability of constraint conformance but was not critical for ensuring separation in overconstrained scenarios. The implementation of priority flight rules through staggered alerting thresholds was completely effective.

- $\mathrm{CD}, \mathrm{CR}$ and CP tools must be enhanced to assist pilots in all possible guidance modes. Broadcasting the commanded trajectory 
rather than an FMS flight plan would reduce unnecessary maneuvering and adverse aircraft interactions resulting from false conflict alerts for the receiving aircraft. These features are being incorporated in the continued development of AOP.

\section{Future Work}

The next step in investigating the feasibility of autonomous aircraft operations is to examine the interactions between autonomous aircraft and the Air Traffic Service provider, and the effect of autonomous aircraft operations on managed aircraft operations. An integrated air/ground simulation experiment is currently being designed by NASA Langley and Ames Research Centers to address these issues. The experiment will involve subject controllers and subject pilots, and will assess the feasibility of autonomous aircraft operations in the arrival flow to a terminal-entry metering fix.

\section{References}

[1] Advanced Air Transportation Technologies Project Office, 1999, Concept Definition for Distributed Air/Ground Traffic Management (DAGTM), Version 1.0, NASA

[2] RTCA Task Force 3, 1995, Final Report of the RTCA Task Force 3: Free Flight Implementation, Washington DC, RTCA, Inc.

[3] Advanced Air Transportation Technologies Project Office, 2002, DAG-TM Concept Element 5 En Route Free Maneuvering Operational Concept Description, NASA

[4] Mondoloni, Stephane, Michael T. Palmer, David J. Wing, 2002, Development of a Prototype Airborne Conflict Detection and Resolution Simulation Capability, AIAA-2002-4446, Reston, VA, AIAA

[5] Ballin, Mark G., Vivek Sharma, Robert A. Vivona, Edward J. Johnson, Ermin Ramiscal, 2002, A Flight Deck Decision Support Tool for Autonomous Airborne Operations, AIAA-2002-4554, Reston, VA, AIAA

[6] RTCA SC-186, 2000, Application of Airborne Conflict Management: Detection, Prevention, \& Resolution, RTCA/DO-263, Washington, DC, RTCA, Inc.
[7] Hoekstra, Jacco M., 2001, Designing for Safety the Free Flight Air Traffic Management Concept, NLR-TP-2001-313, Amsterdam, Netherlands, National Aerospace Laboratory

[8] Kuchar, James K., Lee C. Yang, 1997, Survey of Conflict Detection and Resolution Modeling Methods, AIAA-97-3732, Reston, VA, AIAA

[9] Bilimoria, Karl, Hilda Lee, Aircraft Conflict Resolution with an Arrival Time Constraint, 2002, AIAA-2002-4444, Reston, VA, AIAA

[10] Wing, David J., Bryan E. Barmore, Karthik Krishnamurthy, 2002, Use of Traffic Intent Information by Autonomous Aircraft in Constrained Operations, AIAA-2002-4555, Reston, VA, AIAA

[11] Bilimoria, Karl, Hilda Lee, 2001, Properties of Air Traffic Conflicts for Free and Structured Routing, AIAA-2001-4051, Reston, VA, AIAA

[12] Barmore, Bryan, Edward Johnson, David Wing, Richard Barhydt, 2003, Airborne Conflict Management within Confined Airspace in a Piloted Simulation of DAG-TM Autonomous Aircraft Operations, $5^{\text {th }}$ USA/Europe ATM R\&D Seminar, Budapest, Hungary, http://atm2003.eurocontrol.fr

[13] Barhydt, Richard, Todd Eischeid, Michael Palmer, David Wing, 2003, Regaining Lost Separation in a Piloted Simulation of Autonomous Aircraft Operations, $5^{\text {th }}$ USA/Europe ATM R\&D Seminar, Budapest, Hungary, http://atm2003.eurocontrol.fr

[14] Wing, David, Karthik Krishnamurthy, Richard Barhydt, Bryan Barmore, 2003, Pilot Interactions in an Over-constrained Conflict Scenario as Studied in a Piloted Simulation of Autonomous Aircraft Operations, $5^{\text {th }}$ USA/Europe ATM R\&D Seminar, Budapest, Hungary, http://atm2003.eurocontrol.fr

[15] den Braven, Wim, 1995, Analysis of Aircraft/Air Traffic Control System Performance, AIAA-95-3363, Reston, VA, AIAA

[16] Barhydt, Richard, Anthony W. Warren, 2002, Newly enacted intent changes to ADS-B MASPS Emphasis on operations, compatibility, and integrity, AIAA-2002-4932, Reston, VA, AIAA 